\title{
Sorafenib enhances the antitumor effects of anti-CTLA-4 antibody in a murine cancer model by inhibiting myeloid-derived suppressor cells
}

\author{
TAKANOBU MOTOSHIMA ${ }^{1,2^{*}}$, YOSHIHIRO KOMOHARA ${ }^{2 *}$, HASITA HORLAD $^{2}$, ARIO TAKEUCHI $^{3}$, \\ YOSHIHIRO MAEDA ${ }^{1}$, KENICHIRO TANOUE ${ }^{1}$, YOSHIAKI KAWANO ${ }^{1}$, MAMORU HARADA $^{4}$, \\ MOTOHIRO TAKEYA ${ }^{2}$ and MASATOSHI ETO ${ }^{1}$
}

\begin{abstract}
Departments of ${ }^{1}$ Urology and ${ }^{2}$ Cell Pathology, Graduate School of Medical Sciences, Kumamoto University, Kumamoto; ${ }^{3}$ Department of Urology, Graduate School of Medical Sciences, Kyusyu University, Fukuoka;

${ }^{4}$ Department of Immunology, Shimane University Faculty of Medicine, Izumo, Japan
\end{abstract}

Received January 15, 2015; Accepted March 5, 2015

DOI: $10.3892 /$ or.2015.3893

\begin{abstract}
This antitumor effect of sorafenib is considered to be dependent not only on its direct cytotoxicity to cancer cells but also due to the inhibition of myeloid-derived suppressor cells (MDSCs). Recently, a novel antibody against cytotoxic T-lymphocyte antigen 4 (CTLA-4), which activates lymphocytes, is currently in clinical applications. The aim of the present study was to investigate the synergistic antitumor effects of anti-CTLA-4 antibody (Ab) and sorafenib in a murine cancer model. RENCA cells were subcutaneously inoculated into mice, which were randomly divided into 4 treatment groups: sorafenib plus anti-CTLA- $4 \mathrm{Ab}$, sorafenib plus control $\mathrm{Ab}$, vehicle plus anti-CTLA-4 Ab, and vehicle plus control Ab. Single therapy using anti-CTLA-4 Ab suppressed tumor growth, but no difference was noted when compared with the single therapy group using sorafenib. Notably, the greatest decrease in tumor size was noted with sorafenib plus anti-CTLA-4 Ab (combination therapy), and the highest rate of tumor rejection was observed in the combination therapy group. The number of infiltrating CD4- or CD8-positive lymphocytes was strongly increased in the combination therapy group. These in vivo data indicate that sorafenib increased the immunostimulatory effect of anti-CTLA- $4 \mathrm{Ab}$ even when sorafenib was used at a low dose. An in vitro study using MDSCs and $\mathrm{CD} 8^{+} \mathrm{T}$ cells showed that the inhibitory effect of MDSCs on $\mathrm{CD}^{+} \mathrm{T}$ cells was significantly abrogated
\end{abstract}

Correspondence to: Professor Masatoshi Eto, Department of Urology, Graduate School of Medical Sciences, Kumamoto University, Honjo 1-1-1, Chuo-ku, Kumamoto 860-8556, Japan

E-mail: etom@kumamoto-u.ac.jp

${ }^{*}$ Contributed equally

Key words: anti-CTLA-4 antibody, myeloid-derived suppressor cells, combination therapy, renal cell carcinoma by the combined use of sorafenib and anti-CTLA- $4 \mathrm{Ab}$. Sorafenib suppressed the expression of immunosuppressive factors in MDSCs. These data indicate that combination therapy of sorafenib and anti-CTLA- 4 Ab may be effective in advanced kidney cancer patients.

\section{Introduction}

Renal cell carcinoma (RCC) is a malignant tumor that is resistant to chemotherapy and radiotherapy $(1,2)$. Since no standard biomarkers exist to diagnose kidney cancer, $30 \%$ of kidney cancer patients present with metastatic lesions $(1,2)$. Until recently, effective treatment for metastatic kidney cancer was cytokine-based immunotherapy with interferon- $\alpha$ or interleukin-2, which produce a response rate of only $10-15 \%$ (3).

Sorafenib is a small-molecule tyrosine kinase inhibitor (TKI) for advanced RCC. According to a Japanese survey, the sorafenib response rate and stable disease rate were 21.8-24.0 and 59.9-75.9\%, respectively, and the median progression-free survival was 16.4-37.2 weeks in the sorafenib group (4-6). Although sorafenib has antitumor activities in patients with advanced RCC, this is not curative therapy.

Recently, the beneficial effects of novel immunotherapy using a humanized antibody to cytotoxic T-lymphocyte antigen 4 (CTLA-4, CD152) were reported in patients with malignant melanoma $(7,8)$. CTLA-4 is an inducible receptor expressed by $\mathrm{T}$ cells that ligates the $\mathrm{B} 7$ family of molecules (primarily CD80 and CD86) on antigen-presenting cells $(9,10)$. CTLA-4 activation suppresses proliferation and function of T-lymphocytes, and an antibody to CTLA- 4 abrogates the CTLA-4 signaling pathway. Although objective responses were observed in some patients with ovarian, prostate and kidney cancer, the effect of CTLA-4 antibody has not translated into improved overall long-term survival (11-14).

In many cancer patients, immunosuppression is a major problem for anticancer therapy, and recent studies have indicated that immature myeloid cells or myeloidderived suppressor cells (MDSCs) are associated with immunosuppression in patients with several types of 
malignant tumors $(15,16)$. MDSCs are a heterogeneous population of immature myeloid cells that are defined as

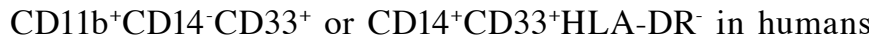
and as $\mathrm{CD} 11 \mathrm{~b}^{+} \mathrm{Grr}^{+}$in mice $(15,16)$. MDSCs assemble in spleen, bone marrow, and tumor tissues in some murine cancer models. In humans, MDSCs circulate in peripheral blood and are occasionally detected in the tumor microenvironment (17). Immunosuppressive mediators expressed in MDSCs include prostaglandin E2, transforming growth factor- $\beta$ (TGF- $\beta$ ), IL-10, indoleamine 2,3-dioxgenase (IDO), nitric oxide synthase 2 (NOS2), and arginase 1 (Arg1) $(18,19)$. Significant reduction in tumor growth was found to be induced by MDSC depletion or inactivation in a murine cancer model (20-22).

Recent findings revealed that sorafenib abrogates the suppressive functions of MDSCs (23). Since MDSCs are considered to limit the efficiency of immunotherapy using anti-CTLA-4 antibody (Ab), we hypothesized that sorafenib may enhance the anticancer effects of anti-CTLA- $4 \mathrm{Ab}$, and thus, in the present study, we tested whether combination therapy is effective in a murine kidney cancer model.

\section{Materials and methods}

Murine cancer model. Animal care protocol and experiments were approved by the Animal Committee at Kumamoto University. RENCA cells $\left(1 \times 10^{6}\right)$, a murine kidney cancer cell line, were subcutaneously inoculated into shaved lateral flanks of 6-8-week-old female BALB/c mice (24). Seven days later, the mice were randomly divided into 4 groups: i) sorafenib (LC Laboratories, Woburn, MA, USA) plus rat antimouse CTLA-4 antibody (UC10-4F10-11; ATCC, Manassas, VA, USA); ii) sorafenib plus control rat immunoglobulin (Santa Cruz Biotechnology, Santa Cruz, CA, USA); iii) vehicle plus anti-CTLA-4 Ab; and iv) vehicle plus control rat immunoglobulin. Sorafenib $(10 \mathrm{mg} / \mathrm{kg})$ or vehicle $(0.5 \%$ methylcellulose) was administered orally once daily for 18 days, and anti-CTLA-4 Ab (100 g/mouse) was administered intraperitoneally every 4 days (Fig. 1A).

Histological analysis and immunostaining. For immunostaining of Ki-67 or proliferating cell nuclear antigen (PCNA), anti-Ki-67 and anti-PCNA antibodies (Dako, Glostrup, Denmark) were used as primary antibodies. For immunostaining of CD4 and CD8, subcutaneous tumor tissues were embedded in OCT compound (Sakura Finetech, Tokyo, Japan), and sections $(5-\mu \mathrm{m})$ that were fixed with cold acetone were treated with the following primary antibodies: anti-CD4 (GK1.5) and anti-CD8 (53-6.72) (both from ATCC). The sections were subsequently treated with HRP-conjugated secondary antibody (Nichirei, Tokyo, Japan). Reactions were visualized with diaminobenzidine (Nichirei). Two investigators who were blinded to any information concerning the samples evaluated infiltration of $\mathrm{CD}^{+}, \mathrm{CD}^{+}, \mathrm{Ki}-67^{+}$and $\mathrm{PCNA}^{+}$cells and the results were averaged as described previously (25).

Lymphocyte proliferation assay. To purify $\mathrm{CD}^{+} \mathrm{T}$ cells, erythrocyte-depleted splenocytes from naïve $\mathrm{BALB} / \mathrm{c}$ mice were incubated with anti-CD8 microbeads and passed over a column according to the manufacturer's instructions (Miltenyi
Table I. Primer list for real-time PCR.

Primer sequences

\begin{tabular}{ll}
\hline TGF- $\beta$ & F: 5'-GCCCTGGATACCAACTATTG-3' \\
& R: 5'-CAGGAGCGCACAATCATGTT-3' \\
Arg1 & F: 5'-CGCCTTTCTCAAAAGGACAG-3' \\
& R: 5'-ACATCAACAAAGGCCAGGTC-3' \\
$\beta$-actin & F: 5'-TCACCCACACTGTGCCCATCT-3' \\
& R:5'-CATCGGAACCGCTCGTTGCCAATA-3' \\
COX2 & F: 5'-TCCTGGAACATGGACTCACTC-3' \\
& R: 5'-CATCTCTCTGCTCTGGTCAATG-3' \\
IDO1 & F: CAGGCCAGAGCAGCATCTTC \\
& R: GCCAGCCTCGTGTTTATTCC \\
IDO2 & F: TGTCCTGGTGCTTAGCAGTCATGT \\
& R: TGCAGGATGTGAACCTCTAACGCT \\
IL-10 & F: CTGCTAACCGACTCCTTAATGC \\
& R: TGAGGGTCTTCAGCTTCTCAC \\
NOS2 & F: CCTTGTTCAGCTACGCCTTC \\
(iNOS) & R: AAGGCCAAACACAGCATACC \\
\hline
\end{tabular}

F, forward; R, reverse; TGF- $\beta$, transforming growth factor- $\beta$; Arg1, arginase 1; COX2, cyclooxygenase 2; NOS2, nitric oxide synthase 2; IDO, indoleamine 2,3-dioxgenase.

Biotec, Bergisch Gladbach, Germany). To purify MDSCs, single-cell suspensions from the spleen of tumor-bearing mice were incubated with anti-Gr-1-biotin Abs, followed by a positive magnetic selection using anti-biotin microbeads according to the manufacturer's instructions (Miltenyi Biotec). The purity of the MDSC population was higher than $90 \%$. Purified $\mathrm{CD}^{+} \mathrm{T}$ cells $\left(5 \times 10^{4} /\right.$ well $)$ from the spleens were cultured in 96-well flat-bottom plates coated with anti-CD3 and anti-CD28 antibodies (both from BioLegends, San Diego, CA, USA). Purified MDSCs $\left(2 \times 10^{5} /\right.$ well) were added to $\mathrm{T}$ cell-cultured plates. MDSCs and T cells were co-cultured for $72 \mathrm{~h}$ in RPMI-1640 supplemented with $8 \%$ fetal bovine serum (Invitrogen, Tokyo, Japan). $\left[{ }^{3} \mathrm{H}\right]$-thymidine was added to the culture $(0.7 \mu \mathrm{Ci} /$ well $)$ during the last $20 \mathrm{~h}$. The cells were harvested onto glass fiber filters, and cell incorporation of $\left[{ }^{3} \mathrm{H}\right]$-thymidine was measured with $\beta$-scintillation counting.

Quantitative real-time PCR. MDSCs were isolated from the tumor-bearing mice, and then cultured with or without sorafenib $(0.125$ and $0.5 \mu \mathrm{M})$ for $24 \mathrm{~h}$. Total RNA was extracted with an RNA STAT-60 extraction kit (Tel-Test, Inc., Friendswood, TX, USA). RNA was reverse transcribed using an ExScript RT reagent kit (Takara Bio, Inc., Ohtsu, Japan). Q-PCR was performed using TaqMan polymerase, and SYBR-Green fluorescence (both from Takara Bio, Inc.) was detected with an ABI PRISM 7300 sequence detector (Applied Biosystems, Foster City, CA, USA). Primers are listed in Table I.

Statistical analysis. Statistical analysis was carried out using StatMate III software (Atoms, Tokyo, Japan). Statistical 
A

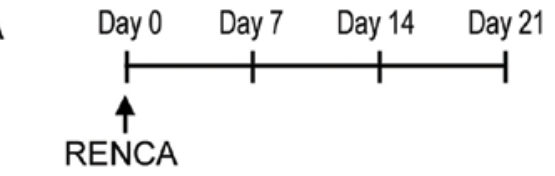

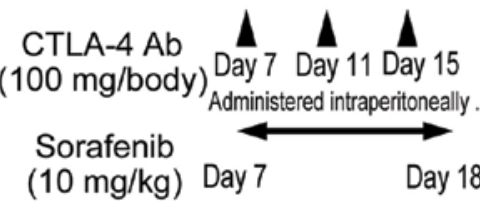

Administered orally once daily.

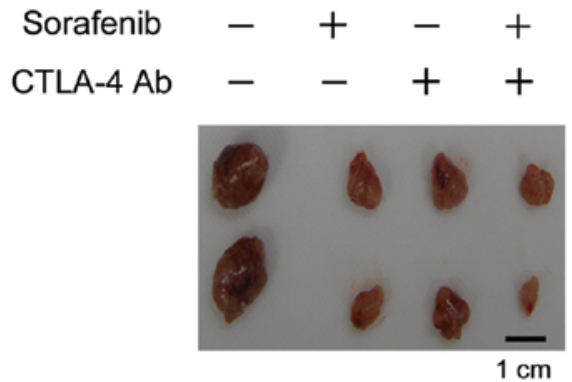

D
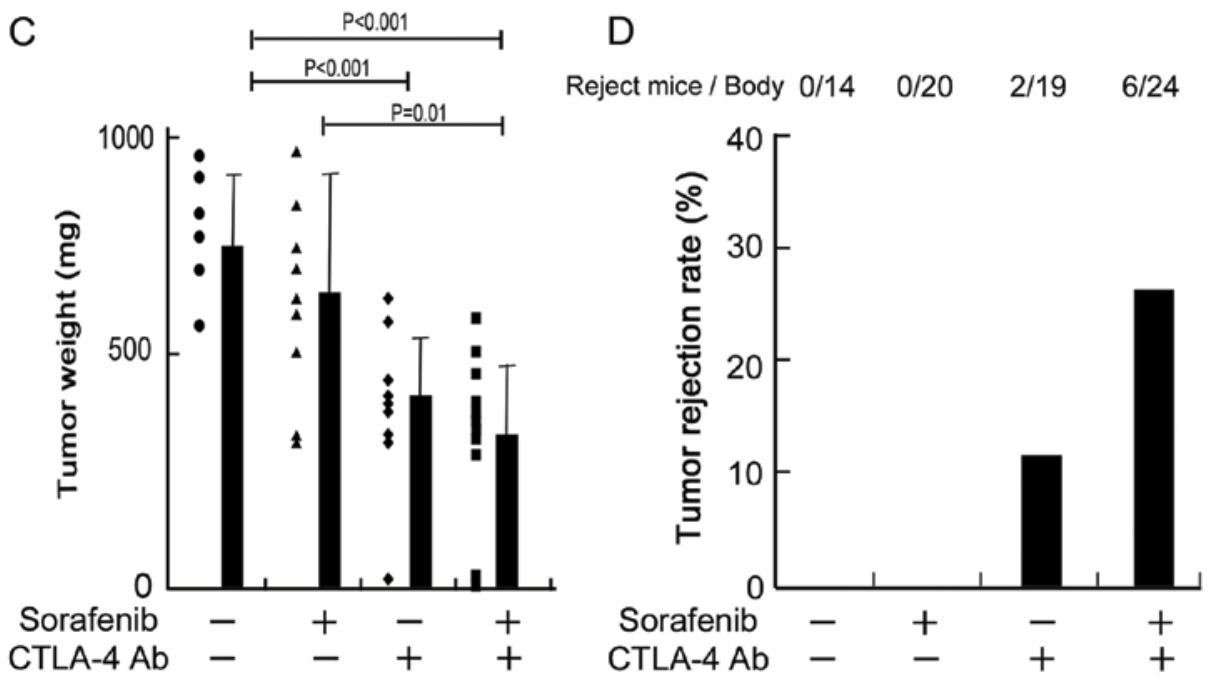

Figure 1. Antitumor effect of sorafenib and CTLA-4 Ab. (A) The protocol for implantation of RENCA cells and treatment with sorafenib and CTLA-4 Ab. Tumor samples were resected (B) and the weight was evaluated (C). (D) The rejection rate of the subcutaneous tumors was calculated. CTLA-4, cytotoxic T-lymphocyte antigen 4.

significance was determined with a Student's t-test or ANOVA test, and $\mathrm{P}<0.05$ was considered to be statistically significant.

\section{Results}

Combination therapy in the RENCA tumor model. We first evaluated the in vivo antitumor efficacy of anti-CTLA-4 Ab combined with sorafenib in a subcutaneous tumor implantation model using RENCA cells. Although the standard protocol for sorafenib treatment in this murine tumor model is $10-50 \mathrm{mg}$ / $\mathrm{kg}$ once a day following tumor cell implantation, the amount of sorafenib administered in the present study was set lower $(10 \mathrm{mg} / \mathrm{kg}$, from 7 to 18 day) to minimize the direct effect of sorafenib on tumor cells. The experimental procedures are shown in Fig. 1A. Although tumor growth in the mice treated with single therapy using sorafenib was slightly suppressed, the difference was not statistically significant when compared to the control group (Fig. 1B and 1C). Since tumor growth was significantly inhibited by single therapy using antiCTLA-4 Ab, no significant differences in tumor size were observed between single therapy using anti-CTLA-4 Ab and the combination therapy (Fig. 1C). However, the rate of tumor rejection was highest in the mice treated with the combination therapy (Fig. 1D).

Histological analysis of tumor samples. Immunostaining for PCNA and Ki-67 was performed to evaluate tumor cell proliferation in non-necrotic areas. The percentage of $\mathrm{PCNA}^{+}$tumor cells was decreased with single therapy using anti-CTLA-4 Ab and the combination therapy (Fig. 2). The percentage of $\mathrm{Ki}-67^{+}$tumor cells was significantly decreased with combination therapy, but no significant change in the percentage of $\mathrm{Ki}-67^{+}$tumor cells was noted in the mice treated with single therapy (Fig. 2).

Lymphocyte infiltration into the tumor samples. Next, we investigated lymphocyte infiltration into the tumor tissues to evaluate the antitumor immune response. Increased numbers of $\mathrm{CD}^{+}$lymphocytes were detected in the mice treated with either single or combination therapy, and importantly, the greatest increase in infiltrated $\mathrm{CD}^{+}$lymphocytes was detected in the mice treated with the combination therapy (Fig. 3). Increased numbers of $\mathrm{CD}^{+}$lymphocytes were detected in the mice treated with anti-CTLA-4 Ab alone or with the combination therapy. However, the greatest increase in the number of infiltrating $\mathrm{CD}^{+}$lymphocytes was detected in the mice treated with the combination therapy. Furthermore, the addition of anti-CTLA-4 Ab to sorafenib significantly increased both $\mathrm{CD}^{+}$and $\mathrm{CD}^{+}$lymphocytes compared with sorafenib alone (Fig. 3). Although there was no statistically significant difference in the numbers of infiltrating lymphocytes between single therapy using anti-CTLA-4 Ab and the combination therapy, the numbers of infiltrating lymphocytes had a tendency to be highest in mice treated with the combination therapy. 

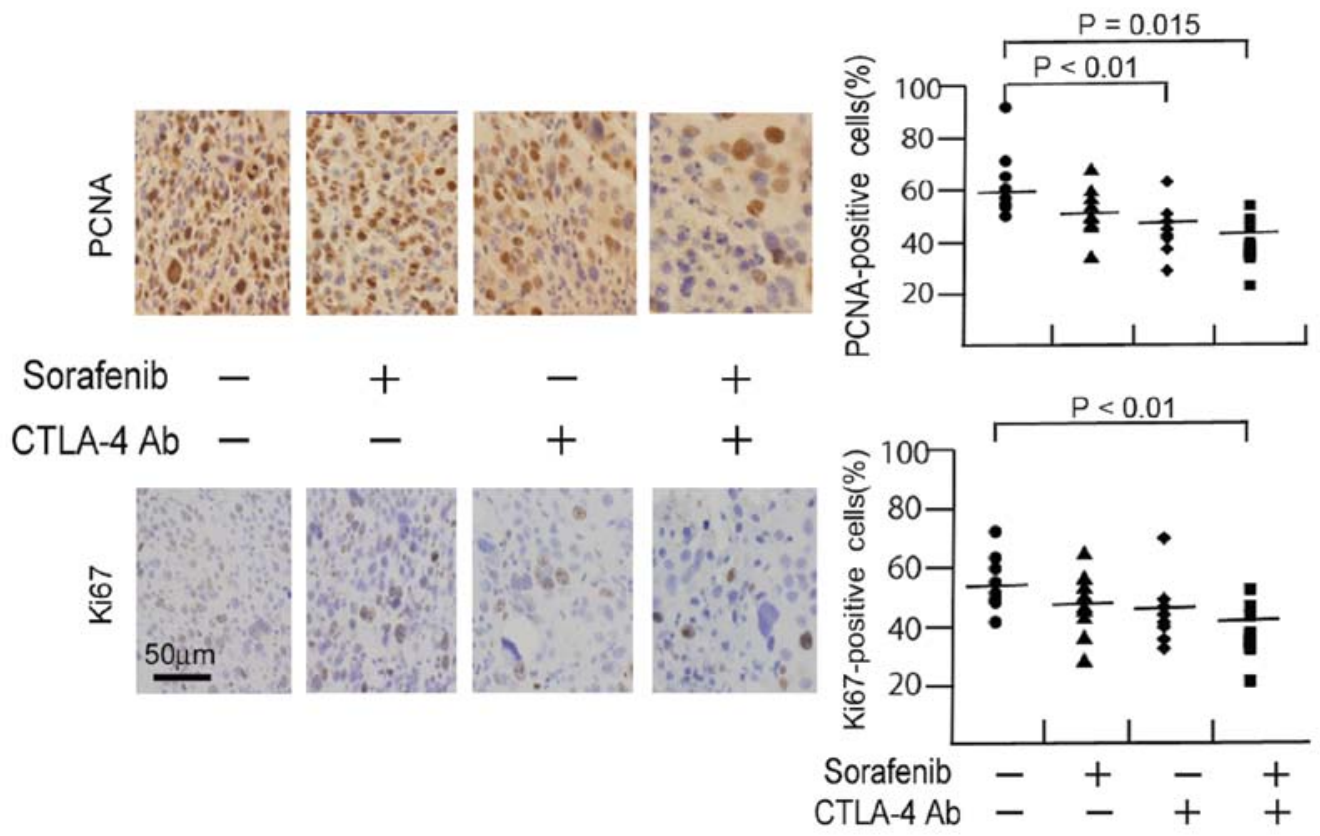

Figure 2. Histological analysis of tumor sections. Immunostaining for PCNA and Ki-67 were performed in the subcutaneous tumors. PCNA, proliferating cell nuclear antigen.
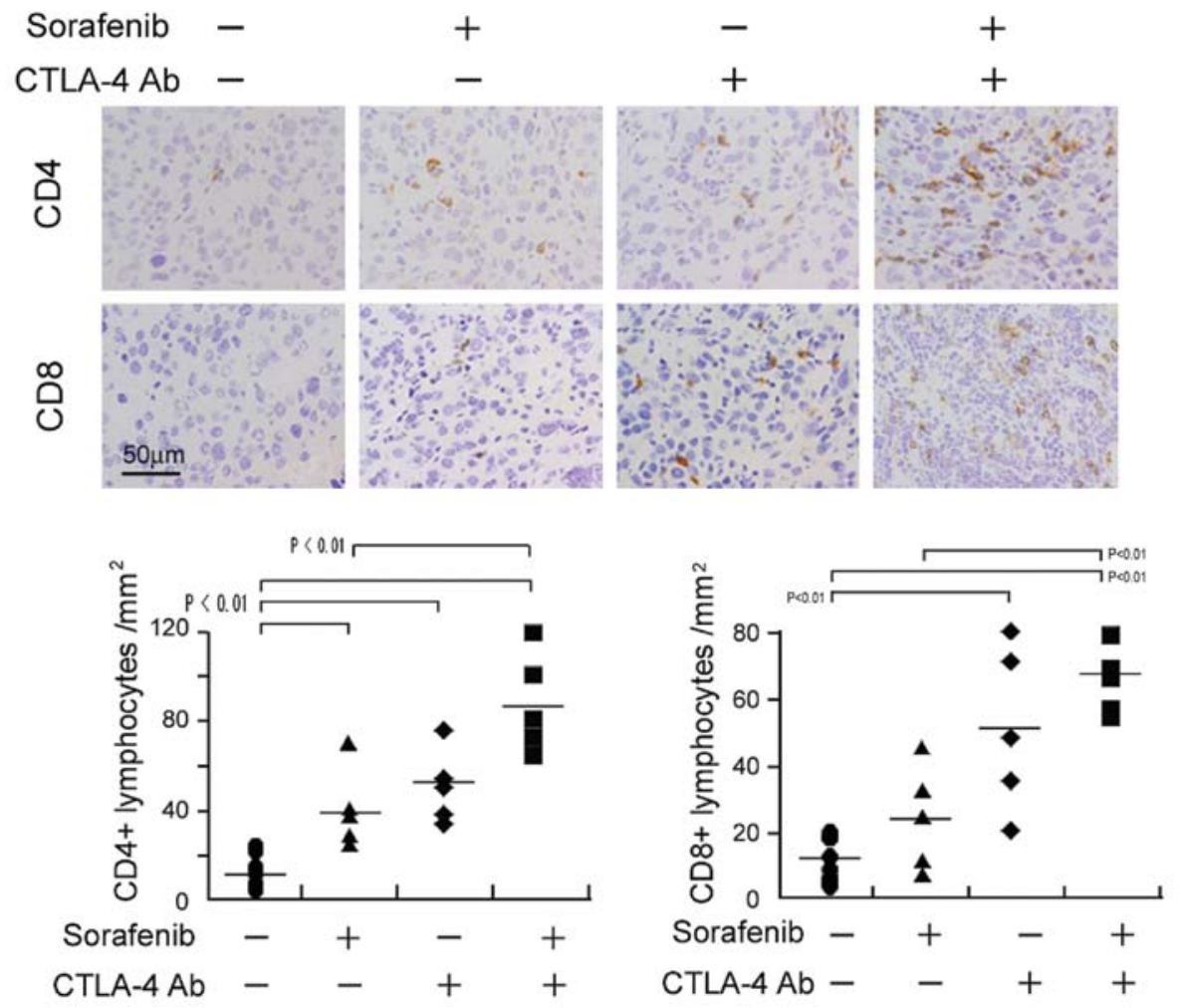

Figure 3. Histological analysis of infiltrating cells in the tumor microenvironment. Immunostaining for CD4 and CD8 was performed to evaluate lymphocyte infiltration, and the cell numbers were counted and statistically analyzed.

The in vitro effect of sorafenib and anti-CTLA-4 Ab on cell-cell interactions between MDSCs and lymphocytes. To investigate whether MDSCs are involved in immune activation due to treatment with sorafenib and anti-CTLA-4 Ab, in vitro analysis was performed using MDSCs isolated from tumor-bearing mice and $\mathrm{CD} 8^{+} \mathrm{T}$ cells isolated from naïve mice (Fig. 4A). As shown in Fig. 4B, proliferation of CD8 ${ }^{+}$ $T$ cells in the absence of MDSCs was not altered by sorafenib and anti-CTLA- $4 \mathrm{Ab}$. Proliferation of $\mathrm{CD}^{+} \mathrm{T}$ cells was significantly suppressed by co-culture with MDSCs as expected, and notably, the suppressive effect of MDSCs on $\mathrm{CD} 8^{+} \mathrm{T}$ cells was significantly reversed by the combined use of sorafenib 


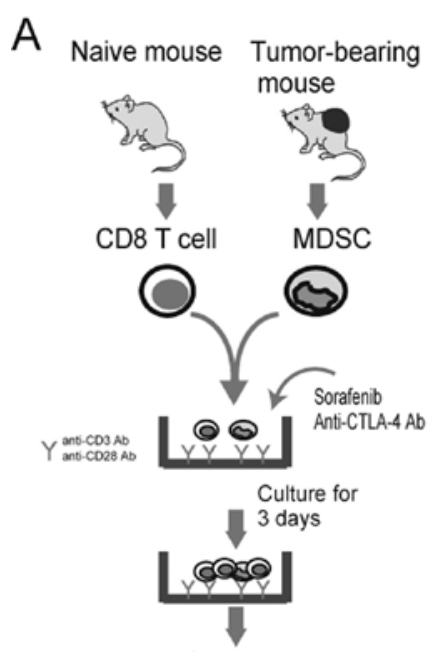

Proliferation assay
B

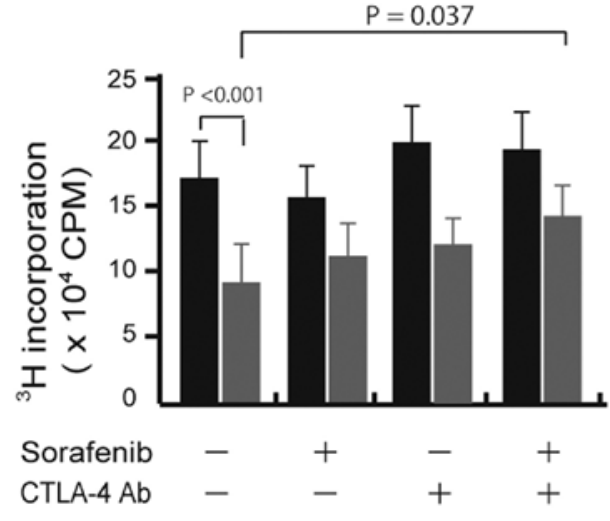

C

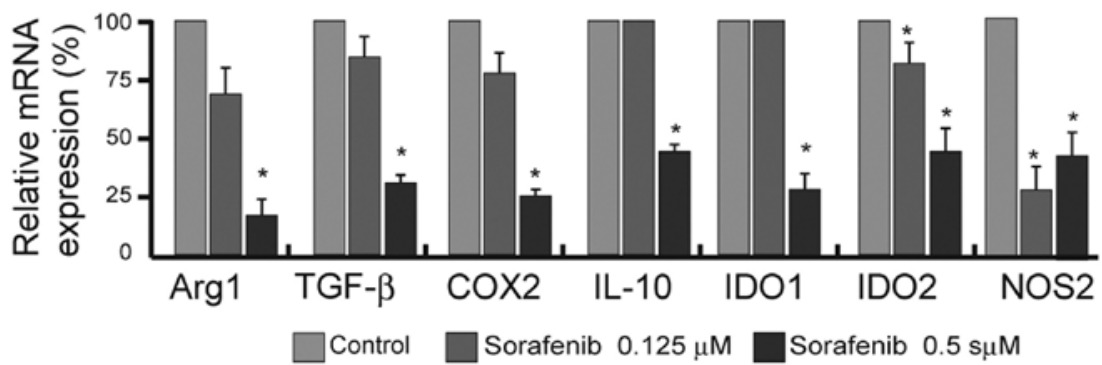

Figure 4. The functional changes in MDSCs by sorafenib and CTLA-4 Ab. (A) A schematic of the in vitro analysis of MDSCs. (B) The influence of sorafenib and CTLA-4 Ab on lymphocyte activation in the non-co-culture condition and in the co-culture condition with MDSCs was evaluated. (C) The mRNA expression of arginase 1 (Arg1), transforming growth factor- $\beta 1$ (TGF- $\beta$ ), cyclooxygenase 2 (COX2), IL-10, indoleamine-2,3-dioxygenase (IDO) 1, IDO2, and NOS2 in MDSCs was evaluated with real-time PCR. "P<0.05. MDSCs, myeloid-derived suppressor cells; CTLA-4, cytotoxic T-lymphocyte antigen 4.

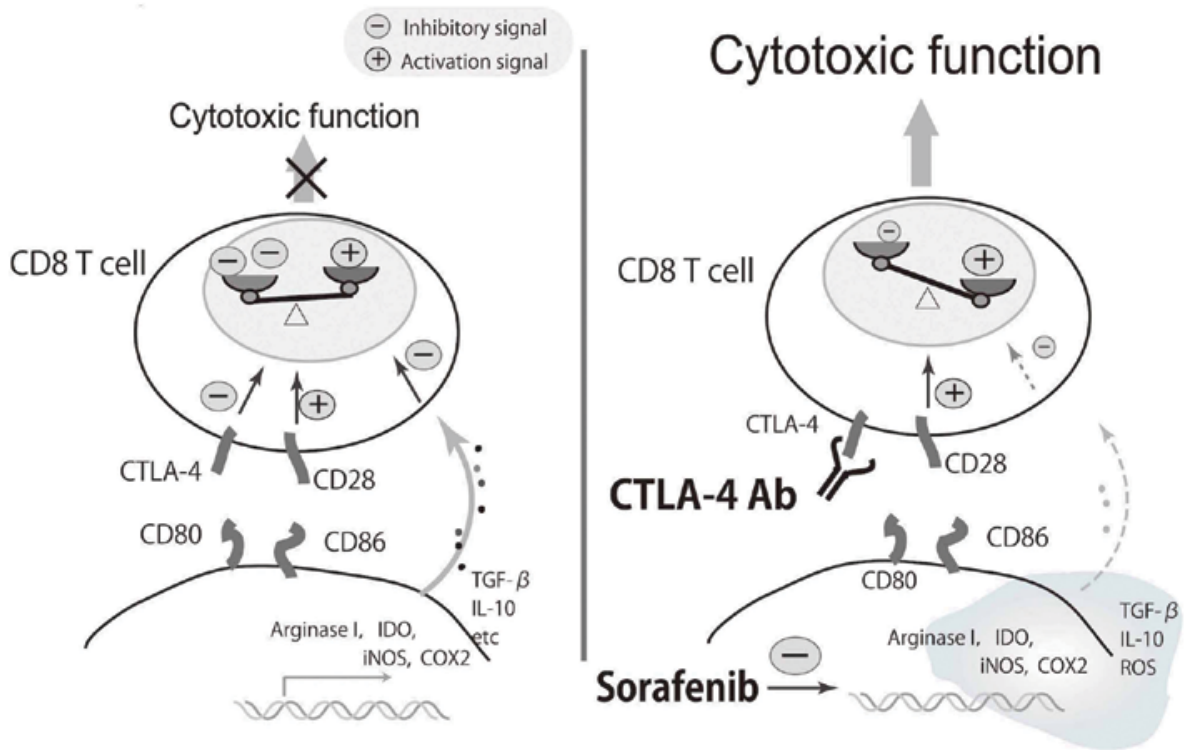

Figure 5. Schematic figure of the synergistic effect of sorafenib and CTLA-4 Ab. Many negative signals are associated with immunosuppression in the tumorbearing host. Sorafenib suppresses production of immunosuppressive factors from MDSCs, and CTLA-4 Ab blocks the CTLA-4-mediated inhibitory signal in lymphocytes. MDSCs, myeloid-derived suppressor cells; CTLA-4, cytotoxic T-lymphocyte antigen 4.

and anti-CTLA-4 Ab (Fig. 4B). No significant reversal in the immunosuppressive effect of MDSCs was induced with single use of either sorafenib or anti-CTLA-4 Ab (Fig. 4B). Next, we tested whether sorafenib suppressed mRNA expression of immunosuppression-related molecules in MDSCs derived from tumor-bearing mice. Real-time PCR analysis showed 
that mRNA expression of Arg1, TGF- $\beta$, cyclooxygenase 2 (COX2), IL-10, IDO1, IDO2, and NOS2 in MDSCs was significantly inhibited by sorafenib (Fig. 4C).

\section{Discussion}

In the present study, we demonstrated that sorafenib enhanced the efficacy of anti-CTLA-4 Ab. The significantly increased number of lymphocytes in tumor tissues induced by the combination therapy indicates that this synergistic effect was mediated by activation of the antitumor immune system (Fig. 5). Although single therapy using low-dose sorafenib increased the number of infiltrating lymphocytes and appeared to activate the antitumor immune system, the antitumor effect of low-dose sorafenib in our in vivo cancer model was not statistically significant in the present study (Fig. 1). Increased efficiency of combination therapy is expected if an increased amount of sorafenib is used in this murine cancer model.

Not only sorafenib but also sunitinib are now widely used as TKIs for the treatment of advanced kidney cancer (26). Both compounds have similar inhibitory effects on kinase receptors such as c-kit, vascular endothelial growth factor receptor (VEGFR), and platelet-derived growth factor receptor (PDGFR). In addition to the direct antitumor effects of both compounds, they also both inhibit the immunosuppressive function of MDSCs (26). Since both compounds have similar inhibitory effects on kinase receptors such as c-kit, VEGFR, and PDGFR $(26,27)$, we used sorafenib in the present study. Further studies are necessary to determine which compound has a stronger influence on MDSCs and shows more synergistic effects with combination therapy.

Currently, much attention is being paid to immune checkpoint blockade using anti-CTLA-4 Ab or anti-programmed death 1 (PD1) Ab (7,28). Ipilimumab, a human IgG1 mAb that blocks CTLA-4, improves overall survival in patients with advanced melanoma $(7,29)$. Nivolumab, a human IgG4 mAb that blocks the PD1 receptor, demonstrates durable objective responses in patients with melanoma and renal cell and non-small cell lung cancer (28). Our present results indicate that ipilimumab in combination with sorafenib may be a treatment option for patients with advanced RCC. Alternatively, an anti-PD1 Ab can also be combined with other TKIs. Such a study is currently underway.

In the present study using cultured MDSCs and $\mathrm{CD}^{+}$ lymphocytes, the proliferation of $\mathrm{CD}^{+}$lymphocytes was significantly suppressed by co-culture with MDSCs. Although only a slight effect was induced by either sorafenib or anti-CTLA-4 Ab alone when CD8 $8^{+}$lymphocytes were cultured without MDSCs, the proliferation of $\mathrm{CD}^{+}$lymphocytes co-cultured with MDSCs was significantly restored by combination use. This indicates that both sorafenib and anti-CTLA- $4 \mathrm{Ab}$ are indispensable for inhibiting the cell-cell interaction between MDSCs and CD8 $8^{+}$lymphocytes.

In conclusion, using in vivo and in vitro preclinical studies, we demonstrated that sorafenib, a multi-kinase receptor inhibitor, enhances anticancer immunotherapy of anti-CTLA- $4 \mathrm{Ab}$. Although immunotherapy using anti-CTLA- $4 \mathrm{Ab}$ is only approved for use in patients with melanoma, anti-CTLA- $4 \mathrm{Ab}$ in combination with sorafenib may be a treatment option for advanced RCC.

\section{Acknowledgements}

We thank Mr. Takenobu Nakagawa, Mr. Osamu Nakamura, Ms. Emi Kiyota and Ms. Yui Hayashida for their technical assistance. This study was supported by grants from the Ministry of Education, Culture, Sports, Science and Technology of Japan. This study was supported in part by a scholarship for the Graduate School of Medical Sciences, Kumamoto University, Japan and by a grant from the Ministry of Education, Science and Culture of Japan (26670703) (to M.E.).

\section{References}

1. Patard JJ, Pignot G, Escudier B, Eisen T, Bex A, Sternberg C, Rini B, Roigas J, Choueiri T, Bukowski R, et al: ICUD-EAU International Consultation on Kidney Cancer 2010: Treatment of metastatic disease. Eur Urol 60: 684-690, 2011.

2. Motzer RJ and Bukowski RM: Targeted therapy for metastatic renal cell carcinoma. J Clin Oncol 24: 5601-5608, 2006.

3. Canil C, Hotte S, Mayhew LA, Waldron TS and Winquist E: Interferon-alfa in the treatment of patients with inoperable locally advanced or metastatic renal cell carcinoma: a systematic review. Can Urol Assoc J 4: 201-208, 2010.

4. Tanigawa G, Kawashima A, Yamaguchi S, Nishimura K, Miyoshi S, Kajikawa J, Meguro N, Yosioka T, Oka T, Hara T, et al; Osaka Renal Cell Carcinoma Clinical Study Collaboration: Clinical outcome and prognostic factors of sorafenib in Japanese patients with advanced renal cell carcinoma in general clinical practice. Jpn J Clin Oncol 41: 1265-1270, 2011.

5. Yuasa T, Tsuchiya N, Urakami S, Horikawa Y, Narita S, Inoue T, Saito M, Yamamoto S, Yonese J, Fukui I, et al: Clinical efficacy and prognostic factors for overall survival in Japanese patients with metastatic renal cell cancer treated with sunitinib. BJU Int 109: 1349-1354, 2012.

6. Naito S, Tsukamoto T, Murai M, Fukino K and Akaza H: Overall survival and good tolerability of long-term use of sorafenib after cytokine treatment: final results of a phase II trial of sorafenib in Japanese patients with metastatic renal cell carcinoma. BJU Int 108: 1813-1819, 2011.

7. Robert C, Thomas L, Bondarenko I, O'Day S, Weber J, Garbe C, Lebbe C, Baurain JF, Testori A, Grob JJ, et al: Ipilimumab plus dacarbazine for previously untreated metastatic melanoma. $\mathrm{N}$ Engl J Med 364: 2517-2526, 2011.

8. Hodi FS, O'Day SJ, McDermott DF, Weber RW, Sosman JA, Haanen JB, Gonzalez R, Robert C, Schadendorf D, Hassel JC, et al: Improved survival with ipilimumab in patients with metastatic melanoma. N Engl J Med 363: 711-723, 2010.

9. Postow MA, Callahan MK and Wolchok JD: The antitumor immunity of ipilimumab: (T-cell) memories to last a lifetime? Clin Cancer Res 18: 1821-1823, 2012.

10. Ascierto PA, Marincola FM and Ribas A: Anti-CTLA4 monoclonal antibodies: the past and the future in clinical application. J Transl Med 9: 196, 2011.

11. Graziani G, Tentori L and Navarra P: Ipilimumab: a novel immunostimulatory monoclonal antibody for the treatment of cancer. Pharmacol Res 65: 9-22, 2012.

12. Hodi FS, Mihm MC, Soiffer RJ, Haluska FG, Butler M, Seiden MV, Davis T, Henry-Spires R, MacRae S, Willman A, et al: Biologic activity of cytotoxic $\mathrm{T}$ lymphocyte-associated antigen 4 antibody blockade in previously vaccinated metastatic melanoma and ovarian carcinoma patients. Proc Natl Acad Sci USA 100: 4712-4717, 2003.

13. Small EJ, Tchekmedyian NS, Rini BI, Fong L, Lowy I and Allison JP: A pilot trial of CTLA-4 blockade with human antiCTLA-4 in patients with hormone-refractory prostate cancer. Clin Cancer Res 13: 1810-1815, 2007.

14. Blansfield JA, Beck KE, Tran K, Yang JC, Hughes MS, Kammula US, Royal RE, Topalian SL, Haworth LR, Levy C, et al: Cytotoxic T-lymphocyte-associated antigen- 4 blockage can induce autoimmune hypophysitis in patients with metastatic melanoma and renal cancer. J Immunother 28: 593-598, 2005.

15. Youn JI and Gabrilovich DI: The biology of myeloid-derived suppressor cells: the blessing and the curse of morphological and functional heterogeneity. Eur J Immunol 40: 2969-2975, 2010.

16. Ostrand-Rosenberg S: Myeloid-derived suppressor cells: more mechanisms for inhibiting antitumor immunity. Cancer Immunol Immunother 59: 1593-1600, 2010. 
17. Gabrilovich DI and Nagaraj S: Myeloid-derived suppressor cells as regulators of the immune system. Nat Rev Immunol 9: 162-174, 2009.

18. Ostrand-Rosenberg S, Sinha P, Beury DW and Clements VK: Cross-talk between myeloid-derived suppressor cells (MDSC) macrophages, and dendritic cells enhances tumor-induced immune suppression. Semin Cancer Biol 22: 275-281, 2012.

19. Bierie B and Moses HL: Transforming growth factor beta (TGF-beta) and inflammation in cancer. Cytokine Growth Factor Rev 21: 49-59, 2010.

20. Porembka MR, Mitchem JB, Belt BA, Hsieh CS, Lee HM, Herndon J, Gillanders WE, Linehan DC and Goedegebuure P: Pancreatic adenocarcinoma induces bone marrow mobilization of myeloid-derived suppressor cells which promote primary tumor growth. Cancer Immunol Immunother 61: 1373-1385, 2012.

21. Fujita M, Kohanbash G, Fellows-Mayle W, Hamilton RL, Komohara Y, Decker SA, Ohlfest JR and Okada H: COX-2 blockade suppresses gliomagenesis by inhibiting myeloid-derived suppressor cells. Cancer Res 71: 2664-2674, 2011.

22. Srivastava MK, Zhu L, Harris-White M, Kar UK, Huang M, Johnson MF, Lee JM, Elashoff D, Strieter R, Dubinett S, et al: Myeloid suppressor cell depletion augments antitumor activity in lung cancer. PLoS One 7: e40677, 2012.

23. Cao M, Xu Y, Youn JI, Cabrera R, Zhang X, Gabrilovich D, Nelson DR and Liu C: Kinase inhibitor sorafenib modulates immunosuppressive cell populations in a murine liver cancer model. Lab Invest 91: 598-608, 2011.
24. Takeuchi A, Eto M, Tatsugami K, Yamada H, Oki T, Kiyoshima K, Yoshikai Y and Naito S: Mechanism of synergistic antitumor effect of sorafenib and interferon- $\alpha$ on treatment of renal cell carcinoma. J Urol 184: 2549-2556, 2010.

25. Horlad H, Fujiwara Y, Takemura K, Ohnishi K, Ikeda T, Tsukamoto $\mathrm{H}$, Mizuta $\mathrm{H}$, Nishimura $\mathrm{Y}$, Takeya $\mathrm{M}$ and Komohara Y: Corosolic acid impairs tumor development and lung metastasis by inhibiting the immunosuppressive activity of myeloid-derived suppressor cells. Mol Nutr Food Res 57: 1046-1054, 2013.

26. Figlin R, Sternberg C and Wood CG: Novel agents and approaches for advanced renal cell carcinoma. J Urol 188: 707-715, 2012.

27. Patel PH, Chaganti RS and Motzer RJ: Targeted therapy for metastatic renal cell carcinoma. Br J Cancer 94: 614-619, 2006.

28. Topalian SL, Hodi FS, Brahmer JR, Gettinger SN, Smith DC, McDermott DF, Powderly JD, Carvajal RD, Sosman JA, Atkins MB, et al: Safety, activity, and immune correlates of anti-PD-1 antibody in cancer. N Engl J Med 366: 2443-2454, 2012.

29. Wolchok JD, Kluger H, Callahan MK, Postow MA, Rizvi NA, Lesokhin AM, Segal NH, Ariyan CE, Gordon RA, Reed K, et al: Nivolumab plus ipilimumab in advanced melanoma. N Engl J Med 369: 122-133, 2013. 